\title{
Bakterielle Proteinsekretion
}

\section{Export von Gefahrgut: Helicobacter pylori und sein CagA-Protein}

CLARA LETTL, WOLFGANG FISCHER

MAX VON PETTENKOFER-INSTITUT FÜR HYGIENE UND MEDIZINISCHE MIKROBIOLOGIE, MEDIZINISCHE FAKULTÄT, LMU MÜNCHEN

Pathogenic bacteria often utilize type IV secretion systems to interact with host cells and to modify their microenvironment in a favourable way. The human pathogen Helicobacter pylori produces such a system to inject only a single protein, CagA, into gastric cells, but this injection represents a major risk factor for gastric cancer development. Here, we discuss the unusual structure of the Cag secretion nanomachine and other features that make it unique among bacterial protein transporters.

DOI: $10.1007 / \mathrm{s} 12268-020-1454-7$

(C) Die Autoren 2020

Bakterielle Infektionen entstehen oft infolge einer Invasion des Krankheitserregers in sterile Körperbereiche oder durch zu starke Vermehrung gegenüber der normalen Mikrobiota. Der Magenkeim Helicobacter pylori ist anders: Er ist meistens außerhalb der Magenschleimhaut zu finden und verdrängt bei der Pathogenese keine anderen Bakterien. Tatsächlich könnte man H. pylori aufgrund seines häufigen Vorkommens und seiner optimalen Anpassung als typische Mikrobiota des menschlichen Magens betrachten, wäre da nicht der Umstand, dass die Kolonisierung stets eine chronische Entzündung der Magenschleimhaut und häufig schwere Komplikationen wie Magengeschwüre oder Magenkrebs verursacht. Ein wichtiger Punkt ist dabei der chronische Verlauf der Infektion: Die „Ansteckung“ erfolgt üblicherweise im Kindesalter, wobei die Bakterien meistens innerhalb der Familie auf die jüngere Generation übertragen werden, während Beschwerden oft erst im fortgeschrittenen Erwachsenenalter auftreten.

Evolutionär gesehen ist $H$. pylori deswegen ein ständiger Begleiter der Menschheit über mindestens die letzten 100.000 Jahre hinweg [1]. In dieser Zeit haben sich weltweit in lokalen Bevölkerungsgruppen Varianten von $H$. pylori herausgebildet. So unterscheiden sich etwa Isolate von Patienten aus Ostasien genetisch von solchen aus Westafrika oder Europa. Tatsächlich konnten große Migra- tionsbewegungen der Menschheitsgeschichte, wie die ursprüngliche Auswanderung aus Ostafrika oder die von Taiwan ausgehende Besiedlung der südpazifischen Inseln anhand der Diversität entsprechender H. pylori-Isolate schlüssig nachverfolgt werden.

Eine Ursache, viele Wirkungen: CagA als Pathogenitätsfaktor

Für die Entstehung schwerer Verläufe sind neben der chronischen Entzündung der Magenschleimhaut (Gastritis) auch bakterielle Pathogenitätsfaktoren verantwortlich. plasmamembran der Zielzelle.
Als entscheidendes Ereignis in der Evolution von $H$. pylori gilt in diesem Zusammenhang die Aneignung eines mobilen genetischen Elements, der cytotoxin-associated gene(cag)Pathogenitätsinsel, vor etwa 60.000 Jahren. Diese Insel codiert zum einen das CagAProtein, das als bakterielles Onkoprotein beschrieben wurde [2], und zum anderen ein Typ-IV-Sekretionssystem (T4SS), das CagA als Effektorprotein erkennt und in Magenzellen translozieren („injizieren“) kann (Abb. 1A). Diese Translokation wiederum führt zu zahlreichen Veränderungen in den betroffenen Zellen, die den Bakterien beispielsweise größere Eisenvorräte liefern, die aber auch zur Konsequenz haben, dass Magengewebe mit größerer Wahrscheinlichkeit transformiert. Zudem reagieren die Epithelzellen mit einer verstärkten Entzündungsantwort auf das Cag-T4SS.

Im Gegensatz zu vielen anderen Bakterien, die oft ein ganzes Arsenal von Proteinen mit jeweils spezifischer Funktion zur Sekretion in Wirtszellen bereithalten, ist CagA das einzige bekannte Effektorprotein des Cag-T4SS. Dafür wechselwirkt es bei geringerer Spezifität mit einer ganzen Reihe zellulärer Proteine. Möglich wird dies durch seine ungewöhnliche C-terminale Region, die intrin-

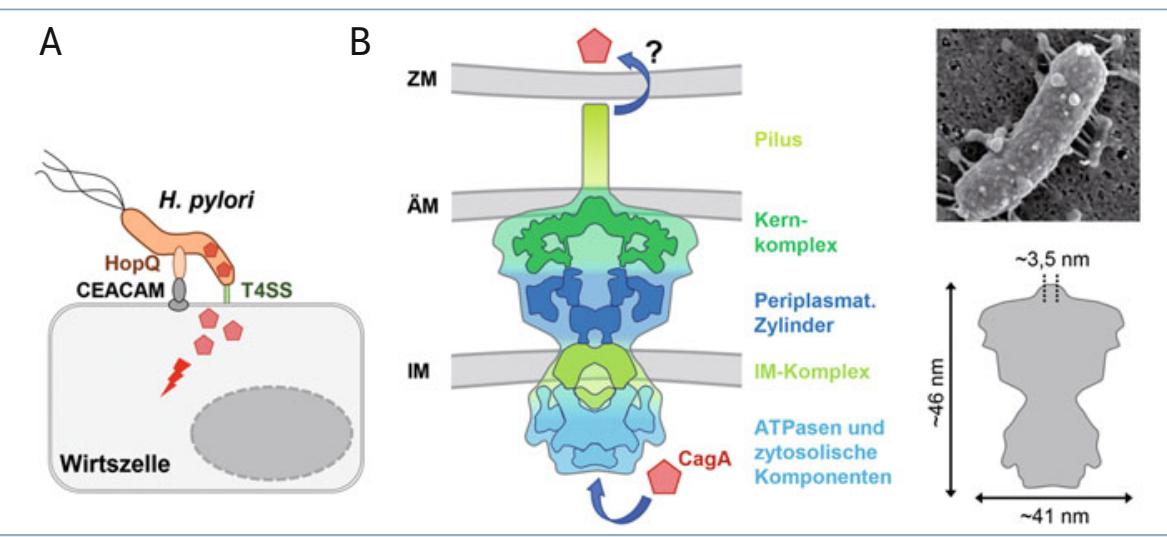

A Abb. 1: Aufbau des Cag-Typ-IV-Sekretionssystems. A, Helicobacter pylori injiziert CagA (rote Fünfecke) über das Cag-T4SS in verschiedene Wirtszellen und nutzt dafür die Bindung seines Membranproteins HopQ an CEACAM-Rezeptoren aus. B, Der Sekretionsapparat ist aus mehreren Subkomplexen aufgebaut und bildet extrazelluläre pilusartige Strukturen aus, die im Elektronenmikroskop erkennbar sind (rechts oben). IM: innere Membran; ÄM äußere Membran; ZM: Zyto- 
sisch ungeordnet ist und mehrere Interaktionsmotive aufweist: Zum einen wird CagA von Kinasen in der Wirtszelle an dem mehrfach vorkommenden Motiv Glu-Pro-Ile-TyrAla (EPIYA-Motiv) phosphoryliert und kann in der Folge an zelluläre Proteine mit SH2Domänen binden, aber eben mit vergleichsweise geringer Bindespezifität. Zum anderen bindet CagA unabhängig von dieser Phosphorylierung an weitere Wirtszellproteine über MARK kinase inhibitor(MKI)-Motive in der gleichen Region.

CagA kann an über 20 zelluläre Proteine binden und so deren Funktion beeinflussen. Viele dieser Proteine sind an Signaltransduktionsvorgängen oder der Kontrolle der Zellpolarität beteiligt, entsprechend gravierend sind die Folgen. Ein Beispiel ist die FehlAktivierung des „kanonischen“ Wnt-Signalwegs über die Stabilisierung von $\beta$-Catenin, die zusammen mit weiteren Veränderungen zu einer epithelial-mesenchymalen Transition, also einer Entdifferenzierung der Epithelzellen, führen kann [3]. Die genaue Abfolge solcher CagA-vermittelter Prozesse im Verlauf der chronischen Infektion, oder auch der Einfluss der anhaltenden Entzündung und die damit verbundene Anhäufung von DNA-Schäden, müssen jedoch in weiteren Studien besser aufgeklärt werden.

\section{Eine Nanomaschine in Übergröße und ein Andockmanöver}

CagA ist mit keinem anderen bekannten Protein verwandt, und auch die am Sekretionsapparat beteiligten Proteine haben zum Teil kaum Ähnlichkeiten zu den typischen Komponenten klassischer T4SS. Außerdem sind etwa zehn zusätzliche Proteine für den Aufbau des Sekretionsapparats notwendig, die es nur in diesem System gibt. Diese Unterschiede spiegeln sich auch in der dreidimensionalen Struktur wider, die nun mit modernen Kryoelektronentomographie-Verfahren weitgehend aufgeklärt werden konnte (Abb. 1B, [4, 5]).

Der im Periplasma und an der äußeren Membran angeordnete Kernkomplex zeigt zwar die typische 14-zählige Rotationssymmetrie, ist aber mit einem Außendurchmesser von $41 \mathrm{~nm}$ deutlich größer als bei den üblichen T4SS mit 17-23 nm; nur der ebenfalls untypische Icm/Dot-Sekretionsapparat von Legionella hat vergleichbare Dimensionen. Das erhöhte Volumen kommt vor allem durch den Einbau zweier Extra-Proteine zustande, aber wohl auch durch zusätzliche Domänen der drei Standardkomponenten.
Über einen periplasmatischen Zylinder ist der Kernkomplex mit einem zweiten großen Komplex in der inneren Membran verbunden, auf dessen zytosolischer Seite die drei für die Funktion benötigten ATPasen in sechsfacher Symmetrie angeordnet sind. Zusammengenommen ist der Sekretionsapparat mindestens 7 MDa schwer und deutlich größer als ein Ribosom. Auf der extrazellulären Seite kann bei Wirtszellkontakt eine pilusartige Struktur aufgebaut werden, die im Elektronenmikroskop darstellbar ist (Abb. 1B), deren Zusammensetzung und Feinstruktur aber nicht genau bekannt sind. Während im Sekretionskomplex ein innerer Kanal zu erkennen ist, durch den CagA aus der Bakterienzelle gelangen könnte, ist derzeit weitgehend unklar, wie sein Transport über die Membran der Zielzelle abläuft.

Man weiß aber, dass Proteine des Sekretionsapparats an Integrin-Rezeptoren auf der Zelloberfläche binden können. Lange Zeit wurde angenommen, dass dies eine wichtige Rolle bei der CagA-Translokation spielt. Neuere Untersuchungen, bei denen mithilfe der CRISPR-Cas-Technologie alle Integrine aus Zielzellen entfernt wurden, konnten jedoch belegen, dass CagA mit unverminderter Effizienz auch in integrinfreie Zellen injiziert werden kann [6]. Im Gegensatz dazu sind Vertreter aus der Familie der $\mathrm{CEACAM}$ (carcinoembryonic antigen-related cell adhesion molecule)-Rezeptoren wesentlich für das Einschleusen von CagA. Die
N-terminalen Domänen dieser CEACAMProteine können über das äußere Membranprotein HopQ, das von allen H. pylori-Stämmen in einer von zwei Varianten produziert wird, zur Anheftung benutzt werden (Abb. 1A, [7]). Die Bindung zwischen HopQ und einem CEACAM-Protein erfolgt dabei auf ähnliche Weise wie die physiologische Bindung zwischen zwei CEACAM-Molekülen, über die eine Adhäsion zwischen benachbarten Zellen vermittelt wird [8]. Wie genau diese Bindung die Typ-IV-Sekretion ermöglicht, ist Gegenstand aktueller Untersuchungen.

\section{Grünes Licht bei Vollsperrung: Anwendungen eines Translokations- Reporters}

Das Cag-T4SS ist wegen seiner exponierten Stellung bei der Pathogenese ein Angriffspunkt für das Immunsystem, wie die verstärkte Entzündungsantwort auf cag-positive H. pylori-Stämme und auch die Bildung von Antikörpern gegen CagA in vielen Patienten zeigen. Ein besseres Verständnis des Sekretionsmechanismus auf molekularer Ebene könnte aber auch zur Entwicklung neuartiger Wirkstoffe gegen $H$. pylori genutzt werden. Zur genaueren Untersuchung des Translokationsvorgangs haben wir kürzlich einen Reporter-Assay entwickelt, der quantitative Messungen des CagA-Transports ermöglicht [9]. Dieser CagA-Translokations-Reporter macht sich die Tatsache zunutze, dass
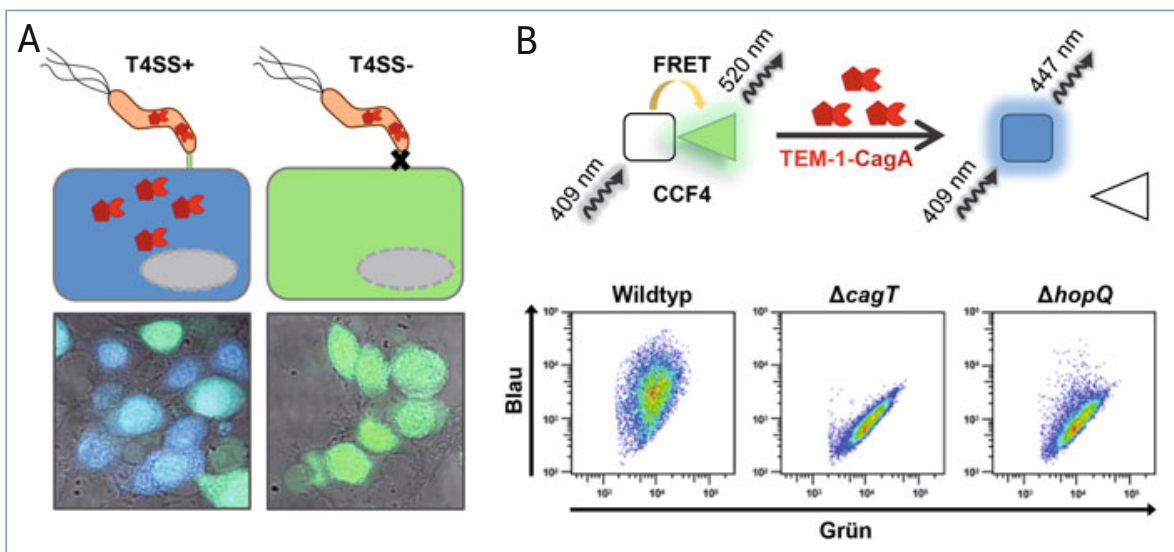

$\Delta$ Abb. 2: Schema des CagA-Translokationsreporters. Helicobacter pylori sekretiert hierbei ein Fusionsprotein aus der $\beta$-Lactamase TEM-1 (roter Mond) und CagA (rotes Fünfeck; links, T4SS+). Intrazelluläres TEM-1 kann via Hydrolyse des $\beta$-Lactams CCF4 gemessen werden, die die beiden angekoppelten Fluorophore trennt und so zu blauer Fluoreszenz führt (rechts oben). Ist die Translokation unterbunden, fluoresziert die Zelle hingegen grün (links, T4SS-). Das Ausmaß der CagATranslokation lässt sich über das Verhältnis von blauer zu grüner Fluoreszenz quantifizieren, etwa per Durchflusszytometrie (rechts unten). Die Translokation von TEM-1-CagA macht sich hier durch eine Verlagerung zu höheren Blauwerten (Wildtyp) bemerkbar, die in Mutanten mit defektem Sekretionsapparat ( $\Delta$ cagT) oder ohne CEACAM-Ligand ( $\triangle h o p Q$ ) nicht oder nur vermindert auftritt. FRET: Förster-Resonanzenergietransfer. 
CagA trotz seiner beachtlichen Größe von 130-140 kDa Fusionen mit fremden Proteindomänen toleriert, die dann als Passagierproteine mit in die Zielzellen transportiert werden. Bei Fusion von CagA mit einer $\beta$-Lactamase (TEM-1) kann so mit dem Cephalosporinderivat CCF4 ein System aufgebaut werden, bei dem in der Zelle ankommendes TEM-1-CagA eine grün fluoreszierende in eine blau fluoreszierende Substanz umsetzt; bei Defekten im Sekretionsapparat bleibt die Zelle dagegen grün (Abb. 2).

Mit diesem Messsystem, das auch für Immunzellen funktioniert [10], kann der Einfluss von T4SS-Proteinen wie CagT oder von indirekt beteiligten Proteinen wie HopQ auf die Typ-IV-Sekretion bestimmt werden (Abb. 2). Auf ähnliche Weise gelang auch eine detaillierte Charakterisierung des C-terminalen Sekretionssignals, über das CagA Zugang zum Sekretionsapparat erhält [9]. Außerdem lässt sich dieses Reportersystem prinzipiell für einen Einsatz im Mikrotiterplatten-Format anpassen. Mit einem solchen miniaturisierten Assay ließen sich systematisch Substanzbanken nach potenziellen Inhibitoren des T4SS durchsuchen, woraus sich wiederum weiterführende Erkenntnisse über dessen molekulare Wirkungsweise, aber auch neue Therapiemöglichkeiten ergeben könnten.

\section{Danksagung}

Wir danken Manfred Rohde für die RasterEM-Aufnahme in Abbildung 1 und Barbara Schmidinger für die DurchflusszytometrieDiagramme in Abbildung 2.

\section{Literatur}

[1] Moodley Y, Linz B, Bond RP et al. (2012) Age of the association between Helicobacter pylori and man. PLoS Pathog 8:e1002693

[2] Hatakeyama M (2014) Helicobacter pylori CagA and gastric cancer: a paradigm for hit-and-run carcinogenesis. Cell Host Microbe 15:306-316

[3] Fischer AS, Sigal M (2019) The role of Wnt and R-spondin in the stomach during health and disease. Biomedicines 7:44 [4] Hu B, Khara P, Song L et al. (2019) In situ molecular architecture of the Helicobacter pylori Cag type IV secretion system. mBio 10:e00849-19

[5] Chung JM, Sheedlo MJ, Campbell AM et al. (2019) Structure of the Helicobacter pylori Cag type IV secretion system. Elife 8:e47644

[6] Zhao Q, Busch B, Jiménez-Soto LF et al. (2018) Integrin but not CEACAM receptors are dispensable for Helicobacter pylori CagA translocation. PLoS Pathog 14:e1007359

[7] Königer V, Holsten L, Harrison U et al. (2016) Helicobacter pylori exploits human CEACAMs via HopQ for adherence and translocation of CagA. Nat Microbiol 2:16233 [8] Bonsor DA, Zhao Q, Schmidinger B et al. (2018) The Helicobacter pylori adhesin protein HopQ exploits the dimer interface of human CEACAMs to facilitate translocation of the oncoprotein CagA. EMBO J 37:e98664

[9] Schindele F, Weiss E, Haas R et al. (2016) Quantitative analysis of CagA type IV secretion by Helicobacter pylori reveals substrate recognition and translocation requirements. Mol Microbiol 100:188-203
[10] Behrens IK, Busch B, Ishikawa-Ankerhold $\mathrm{H}$ et al. (2020) The HopQ-CEACAM interaction controls CagA translocation, phosphorylation, and phagocytosis of Helicobacter pylori in neutrophils. mBio 11:e03256-19

Funding Open Access funding enabled and organized by Projekt DEAL. Open Access Dieser Artikel wird unter der Creative Commons Namensnennun 4.0 International Lizenz veröffentlicht, welche die Nutzung, Vervielfältigung, Bearbeitung, Verbreitung und Wiedergabe in jeglichem Medium und For
erlaubt, sofern Sie den/die ursprünglichen Autor(en) und die Quelle ordnungsgemäß nennen, einen Link zur Creative Commons Lizenz beifügen und angeben, ob Änderungen vorgenommen wurden. Die in diesem Artikel enthaltenen Bilder und sonstiges Drittmaterial unterliegen ebenfalls der genannten Creative Commons Lizenz, sofern sich aus der Abbildungslegende nichts anderes ergibt. Sofern das betreffende Material nicht unter der genannten Creative Commons Lizenz steht und die betreffende Handlung nich Weiterverwendungen des Materials die Einwilligung obes jeweiligen Rechteinhabers einzuholen. Weitere Details zur Lizenz entnehmen Sie bitte der Lizenzinformation auf http://creativecommons org/licenses/by/4.0/deedde.

Korrespondenzadresse:

PD Dr. Wolfgang Fischer

Max von Pettenkofer-Institut für Hygiene und Medizinische Mikrobiologie

Medizinische Fakultät

LMU München

Pettenkoferstraße 9a

D-80336 München

fischer@mvp.Imu.de

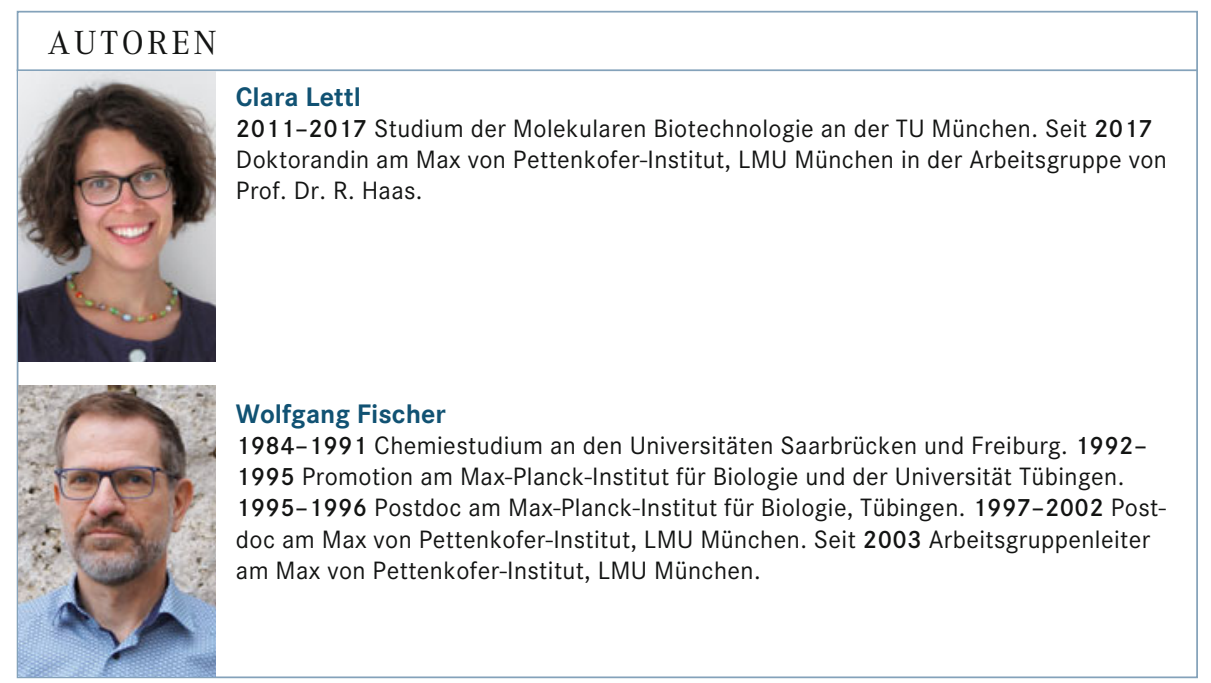

